\title{
SUR UN NOUVEAU MICROPARASITE DU TYPE GRAHAMELLA-RICKETTSIA, OBSERVÉ CHEZ LES POULETS \\ (GRAHAMELLA GALLINARUM)
}

Par M. CARPANO (1)

Chez les poulets, on n'a pas signalé jusqu'ici de parasites du groupe Grahamella-Bartonella-Rickettsia. Dans cette note, nous faisons connaitre nos observations sur un cas que nous avons constaté sur un poulet en nous réservant de revenir sur cette question.

Histoire du cas. - Un éleveur européen présente au Laboratoire de pathologie vétérinaire du Caire, un coq de race Leghorn, élevé en Egypte, gravement malade, dans le but de faire rechercher la nature de la maladie. Il y avait déjà eu des pertes dans le même poulailler.

L'oiseau présentait, parmi d'autres symptòmes, une cyanose intense. L'examen microscopique du sang périphérique fut pratiqué de suite; on constate seulement de rares formes bactérioïdes à coloration bipolaire marquée ; aussi soupçonne-t-on, sur le moment, une infection à Bacillus avisepticum.

On trouve aussi sur cet oiseau, en assez grand nombre, des Argas persicus.

L'animal mourut une vingtaine d'heures après le premier examen. On fit son autopsie pour rechercher la nature des lésions internes et prélever du sang du cœur afin de faire de nouveaux examens microscopiques, des cultures en divers milieux et des inoculations. Au cours de ces recherches, on remarque deux microorganismes particuliers :

$1^{\circ}$ un germe polymorphe endoglobulaire et endocellulaire, voisin des parasites des genres Grahamella, Bartonella, Rickettsia;

$2^{\circ}$ une bactérie présentant les caractères généraux du groupe typho-coli.

(1) Traduit de l'italien par le Docteur Maurice Langeron.

Annales de Parasitologie, $\mathrm{T}$. XIII, $\mathrm{N}^{\circ} 3 .-1^{\text {er }}$ mai 1935 , p. 238-242. 


\section{FoRmes DU TYPE Grahamella}

Ces formes sont représentées par les figures $1,2,3,4$, de la planche V. On les trouvait surtout dans environ 3 p. 100 des érythrocytes. On a remarqué aussi, toujours dans le sang prélevé dans le cœur, des éléments granulaires, inclus dans la partie protoplasmique des grands mononucléaires et de quelques cellules endothéliales.

Les formes trouvées à l'intérieur des hématies avaient une morphologie très variable, allant de la forme coccoïde à celle d'une coccobactérie plus ou moins allongée, ou encore des formes en clou, ou filamenteuses ou ramifiées.

Les dimensions étaient aussi très variables : les formes granulaires étaient excessivement petites, à la limite de la visibilité, on peut même les considérer vraisemblablement comme des éléments filtrables; on arrive ensuite graduellement à des formes plus volumineuses, pouvant atteindre $2-3 \mu$. Le nombre des parasites par hématie est très variable, depuis quelques unités jusqu'à une centaine. Dans ce dernier cas, ils sont souvent très petits.

Ces corpuscules se colorent assez difficilement. Le Giemsa leur donne une teinte rouge violacé qui va jusqu'au rouge rubis de la chromatine chez les petits éléments granulaires. Ces corpuscules prennent le Gram.

L'hématie est sensiblement altérée, surtout quant à l'aspect du noyau. Celui-ci, dans les hématies parasitées, devient plus petit ou perd sa colorabilité. En outre il se déplace vers le bord de l'hématie. La destruction des hématies parasitées met les corpuscules en liberté dans le plasma, comme le montre la figure 3 .

On observe, dans l'ensemble, un véritable processus schizogonique. Les éléments parasitaires observés dans quelques leucocytes et aussi dans quelques cellules endothéliales, sont épars dans la partie protoplasmique des cellules et ressemblent, par leur morphologie générale, aux Rickettsia.

Ils ont une forme arrondie et leurs dimensions ne dépassent pas en moyenne le demi-micron, mais ils peuvent aussi être très petits et atteindre les limites de la visibilité - et par suite être-vraisemblablement filtrables. Ils sont entourés d'un halo et souvent réunis en diplocoques.

Dans les préparations colorées au Giemsa, ils prennent une teinte bleue et ils ne prennent pas le Gram.

La destruction de ces cellules parasitées met en liberté dans le plasma les corpuscules qu'elles renferment; ces derniers cas peu- 
vent aussi être interprétés comme des processus schizogoniques (planche V, fig. 4).

Les formes du type Grahamella n'ont pu être reproduites sur les poulets indigènes inoculés avec le sang de l'oiseau en question. Toutefois les expériences continuent avec les cultures des formes bactéroïdes.

\section{Formes baCtÉroÏDES}

La bactérie isolée, qui se trouvait à l'état pur dans le sang de l'animal original, possède les caractères généraux du groupe typhique-coli. Elle est très peu mobile. Elle se colore facilement avec les colorants ordinaires en solution hydro-alcoolique et ne prend pas le Gram. Elle se présente, en général, sous la forme coccoïde de cocco-bactérie ou de bacille allongé ; elle est extrêmement polymorphe. Souvent elle présente une coloration bipolaire et, traitée par notre méthode de coloration des cils, elle laisse voir un flagelle unique polaire comme le bacille du choléra humain ou le pyocyanique.

\section{EXPLICATION DE LA PLANCHE V}

Fig. 1. - Grahamella gallinarum dans le sang du cœur. Coloration au Giemsa. $\times .1000$. Deux hématies remplies de Grahamella; dans l'une le noyau a perdu sa colorabilité.

Fit. 2.-Grahamella gallinarum dans le sang du cœur. Coloration au Giemsa. $\times$ 1.000. Grands éléments de Grahamella en forme de cocco-bactéries dans une hématie dont le noyau est consirérablement atrophié.

Fig. 3. - Grahamelia gallinarum dans le sang du cour. Coloration au Giemsa. $\times 1.000$ Destruction de l'hématic parasitée dont il reste le novau et mise en liberté de nombreux éléments de Grahamella à morphologie très variée.

Fig. 4. - Préparation de sang du cœur. Coloration au Giemsa. $\times 1.400$. Débris de cellule endothéliale dont la destruction a mis en liberté les éléments granulaires en forme de cocei ou de diplocoques dont quelques-uns sont très petits. Ces éléments sont entourés d'un halo bien net et leur aspect rappelle celui des Rickettsia.

Vic. 5. - Culture sur gélose faite avec le sang du cœur du poulet infecté. Aspect du liquide de condensation après un séjour de 48 heures à l'étuve. Coloration au Giemsa. $\times 1.000 .1^{\circ}$ Formes bactéroïdes fortement colorées en violet, souvent avec coloration bipolaire; $2^{\circ}$ Formes coccoïdes souvent réunies en grappes, légèrement colorées en bleu. Ces derniers áléments, qui n'ont pu être retrouvés dans les repiquages, sont pour nous en relation avee les formes du type Rickettsia contenues dans le protoplasma des cellules endothéliales et des macrophages observés dans le sang du cour.

Fı. 6. - Culture de 24 heures sur gélose de la bactérie isolée du sang du cour. Coloration spéciale pour les eils. $\times 1.000$. Noter le cil polaire unique qui rappelle tout à fait la morphologie du bacille du choléra et du bacille pyocyanique. 

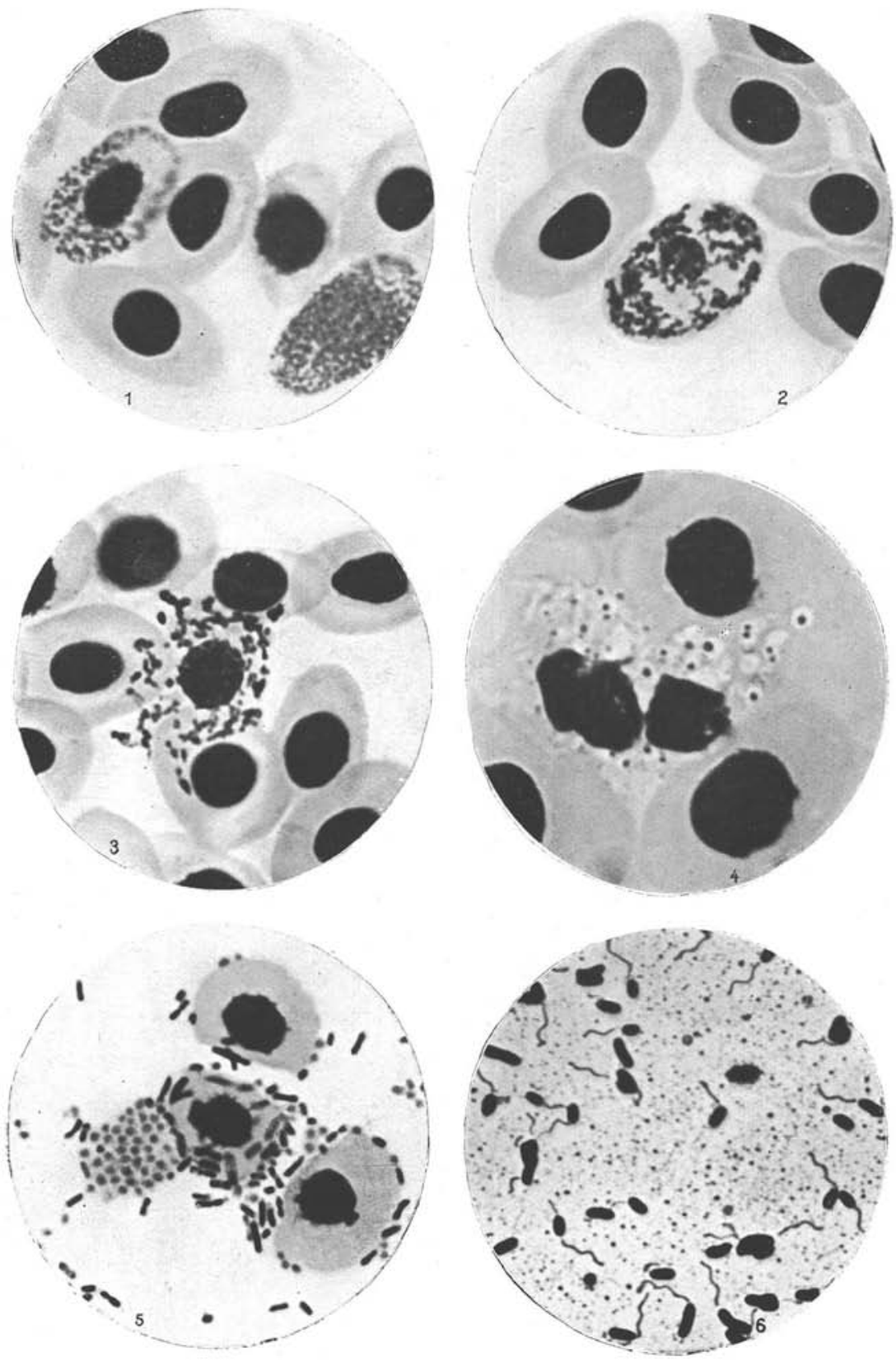
Les cultures ressemblent à celles des bactéries du groupe typhique-coli. Sur bouillon, elles dégagent une légère odeur fétide. La gélatine n'est pas liquéfiée. Le lait est rapidement coagulé et ensuite en partie digéré. Sur pomme de terre, il se produit une patine jaune clair. Il y a fermentation, avec production de gaz, avec les sucres suivants : glycose, maltose, lactose, saccharose, mannite, dextrine, dulcite et xylose.

Cette bactérie est très pathogène, non seulement pour le poulet, mais aussi pour le cobaye, le lapin et le chien, chez lesquels elle détermine une septicémie toujours mortelle.

Chez les animaux inoculés expérimentalement avec ce germe, on n'observe pas la reproduction d'éléments intraglobulaires du type Grahamella décrits plus haut. Les expériences n'ont d'ailleurs été tentées jusqu'ici qu'avec des volatiles indigènes.

En outre, on a trouvé, dans les cultures premières sur gélose au sang, des éléments coccoïdes mesurant environ un demi-micron, coiorés en bleu pâle avec ébauche de structure interne et réunis en grappe (planche V, fig. 5). Il n'a pas été possible de les reproduire dans les cultures secondes, sur le même milieu (gélose au sang de poulet).

Ces formes sont vraisemblablement en rapport avec les éléments trouvés dans les macrophages et dans les cellules endothéliales du sang du cocur. Je les rapproche des Rickettsia à cause de leur morphologie spéciale.

Le fait de trouver en mème temps ces divers agents a grandement compliqué le diagnostic de l'affection dont l'oiseau est mort.

Sont-ce deux parasites complètement différents et réunis accidentellement chez le même animal ? Y a-t-il entre eux un lien de dépendance et font-ils partie du même cycle parasitaire ? L'infection bactérienne surajoutée a-t-elle été capable de réveiller le Grahamella qui existait à l'état latent, comme on l'observe fréquemment dans ce pays pour les infections latentes à hémoprotozoaires ?

Indubitablement, ce cas intéressant fait partie du groupe des infections dans lesquelles des corps intracellulaires, dont la nature n'est pas encore connue et auxquels on a donné les noms de Grahamella, Bartonella, Rickettsia, se trouvent en relation avec des formes bactériennes plus ou moins précises, dans les affections au cours desquelles on a pu constater, dans certains cas, un stade filtrant de l'agent pathogène.

La verruga peruviana et la fièvre de Oroya, le typhus exanthématique, la fièvre des Montagnes Rocheuses et la fièvre des tran- 
chées pour l'homme et, selon quelques auteurs, l'hydropéricardite infectieuse (heartwater) des bovins, des ovins et des caprins en sont des exemples.

Le rapport de notre cas avec ces affections devient encore plus étroit si on ajoute que sur le poulet en question il a été constaté la présence de nombreux Argas persicus qu'on peut soupçonner d'être l'ectoparasite transmetteur.

Le fait de n'avoir pu reproduire expérimentalement les corpuscules intraglobulaires et intracellulaires sur des poulets indigènes et sur d'autres animaux inoculés nous a empêché de procéder à des recherches qui nous auraient permis d'exprimer une opinion plus précise. Nous proposons donc de donner provisoirement à ces corpuscules le nom de Grahamella gallinarum n. sp.

Le Caire, Ministère de l'Agriculture, service vétérinaire. 